\title{
Type transition of simple random walks on randomly directed regular lattices ${ }^{1}$
}

\author{
Massimo CAMPANINo $^{a}$ and Dimitri Petritis ${ }^{b}$ \\ a. Dipartimento di Matematica, Università degli Studi di Bologna, \\ piazza di Porta San Donato 5, I-40126 Bologna, Italy, massimo.campanino@unibo.it \\ b. Institut de Recherche Mathématique, Université de Rennes I and CNRS UMR 6625 \\ Campus de Beaulieu, F-35042 Rennes Cedex, France, dimitri.petritis@univ-rennes1.fr
}

27 November 2018 at 19:44

\begin{abstract}
Simple random walks on a partially directed version of $\mathbb{Z}^{2}$ are considered. More precisely, vertical edges between neighbouring vertices of $\mathbb{Z}^{2}$ can be traversed in both directions (they are undirected) while horizontal edges are one-way. The horizontal orientation is prescribed by a random perturbation of a periodic function, the perturbation probability decays according to a power law in the absolute value of the ordinate. We study the type of the simple random walk, i.e. its being recurrent or transient, and show that there exists a critical value of the decay power, above which it is almost surely recurrent and below which it is almost surely transient.
\end{abstract}

\section{Introduction}

\subsection{Motivations}

We study simple symmetric random walks (i.e. jumping with uniform probability to one of the available neighbours of a given vertex) on partially directed

\footnotetext{
${ }^{1}$ Supported in part by the Italian national PRIN project Random fields, percolation, and stochastic evolution of systems with many components and by the Actions Internationales programme of the Université de Rennes 1. M.C. acknowledges support from G.N.A.M.P.A. This work has been completed while D.P. was on sabbatical leave from his host university at the Institut Henri Poincaré.

2010 Mathematics Subject Classification: 60J10, 60K15

Key words and phrases: Markov chain, random environment, recurrence criteria, random graphs, directed graphs.
} 
regular sublattices of $\mathbb{Z}^{2}$ obtained from $\mathbb{Z}^{2}$ by imposing horizontal lines to be uni-directional. Although random walks on partially directed lattices have been introduced long-time ago to study the hydrodynamic dispersion of a tracer particle in a porous medium [15] very little was known on them beyond some computer simulation heuristics [19]. Therefore, it arose as a surprise for us that so little was rigourously known when we first considered simple random walks on partially directed 2-dimensional lattices in [3, 4]. In those papers, we determined the type of simple random walks on lattices obtained from $\mathbb{Z}^{2}$ by keeping vertical edges bi-directional while horizontal edges become one-way. Depending on how the horizontal allowed direction to the left or the right is determined we obtain dramatically different behaviour [3, Theorems 1.6, 1.7, and 1.8] (these results are reproduced — for completeness — as theorem 1.3 in the present paper).

This result triggered several developments by various authors. In [12], the orientation is chosen by means of a correlated sequence or by a dynamical system; in both cases, provided that some variance condition holds, almost sure transience is established and, in [13], a functional limit theorem is obtained. In [16], the case of orientations chosen according to a stationary sequence is treated. In [17], our results of [3, 4] are used to study corner percolation on $\mathbb{Z}^{2}$. In [7], the Martin boundary of these walks has been studied for the models that are transient and proved to be trivial, i.e. the only positive harmonic functions for the Markov kernel of these walks are the constants. In [8] a model where the horizontal directions are chosen according to an arbitrary (deterministic or random) sequence but the probability of performing a horizontal or vertical move is not determined by the degree but by a sequence of non-degenerate random variables is considered and shown to be a.s. transient.

It is worth noting that all the previous directed lattices are regular in the sense that both the inward and the outward degrees are constant (and equal to 3) all over the lattice. Therefore, the dramatic change of type is due only to the nature of the directedness. However, the type result was always either recurrent or transient. The present paper provides an example where the type of the random walk is determined through the tuning of a parameter controlling the overall number of defects; it improves thus the insight we have on those non reversible random walks. Let us mention also that beyond their theoretical interest (a short list of problems remaining open in the context of such random walks is given in the conclusion section), directed random walks are much more natural models of propagation on large networks like internet than reversible ones. 


\subsection{Notation and definitions}

Directed versions of $\mathbb{Z}^{2}$ are obtained as follows: let $\mathbf{u}=\left(u_{1}, u_{2}\right)$ and $\mathbf{v}=\left(v_{1}, v_{2}\right)$ be arbitrary elements of $\mathbb{Z}^{2}$ and suppose that a sequence of $\{-1,1\}$-valued variables $\boldsymbol{\varepsilon}=\left(\varepsilon_{y}\right)_{y \in \mathbb{Z}}$ is given. The pair $(\mathbf{u}, \mathbf{v}) \in \mathbb{Z}^{2} \times \mathbb{Z}^{2}$ is an allowed edge to the lattice if either $\left[u_{2}=u_{1}\right] \wedge\left[v_{2}=v_{1} \pm 1\right]$ or $\left[v_{2}=v_{1}\right] \wedge\left[u_{2}=u_{1}+\varepsilon_{\nu_{1}}\right]$. The directed sublattice of $\mathbb{Z}^{2}$ depends obviously of the choice of the sequence $\varepsilon$; we denote this partially directed lattice by $\mathbb{Z}_{\boldsymbol{\varepsilon}}^{2}$. The choice of $\boldsymbol{\varepsilon}$ can be deterministic or random and will be specified later.

Definition 1.1. A simple random walk on $\mathbb{Z}_{\varepsilon}^{2}$ is a $\mathbb{Z}^{2}$-valued Markov chain $\left(\mathbf{M}_{n}\right)_{n \in \mathbb{N}}$ with transition probability matrix $P$ having as matrix elements

$$
P(\mathbf{u}, \mathbf{v})=\mathbb{P}\left(\mathbf{M}_{n+1}=\mathbf{v} \mid \mathbf{M}_{n}=\mathbf{u}\right)= \begin{cases}\frac{1}{3} & \text { if }(\mathbf{u}, \mathbf{v}) \text { is an allowed edge of } \mathbb{Z}_{\boldsymbol{\varepsilon}}^{2}, \\ 0 & \text { otherwise }\end{cases}
$$

Remark 1.2. The Markov chain $\left(\mathbf{M}_{n}\right)_{n \in \mathbb{N}}$ cannot be reversible. Therefore, all the powerful techniques based on the analogy with electrical circuits (see $[9,20]$ for modern exposition) or spectral properties of graph Laplacians ${ }^{2}[2,6,5]$ do not apply.

Several $\boldsymbol{\varepsilon}$-horizontally directed lattices have been introduced in [3], where the following theorem has been established.

Theorem 1.3. [3, see theorems 1.6, 1.7, and 1.8] Consider a $\mathbb{Z}_{\boldsymbol{\varepsilon}}^{2}$ directed lattice.

1. If the lattice is alternatively directed, i.e. $\varepsilon_{y}=(-1)^{y}$, for $y \in \mathbb{Z}$, then the simple random walk on it is recurrent.

2. If the lattice has directed half-planes i.e. $\varepsilon_{y}=-\mathbb{1}_{]-\infty, 0[}(y)+\mathbb{1}_{[0, \infty[}(y)$, then the simple random walk on it is transient.

3. If $\varepsilon$ is a sequence of $\{-1,1\}$-valued random variables, independent and identically distributed with uniform probability, the simple random walk on it is transient for almost all possible choices of the horizontal directions.

Notice that the above simple random walks are defined on topologically nontrivial directed graphs in the sense that $\lim _{N \rightarrow \infty} \frac{1}{N} \sum_{y=-N}^{N} \varepsilon_{y}=0$. For the two first cases, this is shown by a simple calculation and for the third case this is an almost sure statement stemming from the independence of the sequence $\boldsymbol{\varepsilon}$. The above condition guarantees that transience is not a trivial consequence of a non-zero drift but an intrinsic property of the walk in spite of its jumps being statistically symmetric.

\footnotetext{
${ }^{2}$ Connections of graph Laplacians with electric circuits is at least as old as reference [21], connections with modern cohomology can be found in $[20,22,14]$ but again ideas are much older $[10,11]$ and references therein.
} 


\subsection{Results}

In this paper, we consider again a $\mathbb{Z}_{\boldsymbol{\varepsilon}}^{2}$ lattice but the sequence $\boldsymbol{\varepsilon}$ is specified as follows.

Definition 1.4. Let $f: \mathbb{Z} \rightarrow\{-1,1\}$ be a $Q$-periodic function with some even integer $Q \geq 2$ verifying $\sum_{y=1}^{Q} f(y)=0$ and $\boldsymbol{\rho}=\left(\rho_{y}\right)_{y \in \mathbb{Z}}$ a Rademacher sequence, i.e. a sequence of independent and identically distributed $\{-1,1\}$-valued random variables having uniform distribution on $\{-1,1\}$. Let $\boldsymbol{\lambda}=\left(\lambda_{y}\right)_{y \in \mathbb{Z}}$ be a $\{0,1\}$ valued sequence of independent random variables and independent of $\boldsymbol{\rho}$ and suppose there exist constants $\beta$ (and $c$ ) such that $\mathbb{P}\left(\lambda_{y}=1\right)=\frac{c}{|y|^{\beta}}$ for large $|y|$. We define the horizontal orientations $\boldsymbol{\varepsilon}=\left(\varepsilon_{y}\right)_{y \in \mathbb{Z}}$ through $\varepsilon_{y}=\left(1-\lambda_{y}\right) f(y)+\lambda_{y} \rho_{y}$. Then the $\mathbb{Z}_{\boldsymbol{\varepsilon}}^{2}$-directed lattice defined above is termed a randomly horizontally directed lattice with randomness decaying in power $\beta$.

Theorem 1.5. Consider the horizontally directed lattice $\mathbb{Z}_{\boldsymbol{\varepsilon}}^{2}$ with randomness decaying in power $\beta$.

1. If $\beta<1$ then the simple random walk is transient for almost all realisations of the sequence $\left(\lambda_{y}, \rho_{y}\right)$.

2. If $\beta>1$ then the simple random walk is recurrent for almost all realisations of the sequence $\left(\lambda_{y}, \rho_{y}\right)$.

Remark 1.6. It is worth noting that the periodicity of the function $f$ is required only to prove recurrence; for proving transience, any function $f$ can be used.

Remark 1.7. In the previous model, the levels $y$ where $\lambda_{y} \neq 0$, can be viewed as random defects perturbing a periodically directed model whose horizontal directions are determined by the periodic function $f$. Thus, it is natural to consider the random variable $\|\boldsymbol{\lambda}\|:=\|\boldsymbol{\lambda}\|_{1}=\operatorname{card}\left\{y \in \mathbb{Z}: \lambda_{y}=1\right\}$ as the strength of the perturbation. When $\beta>1$, by Borel-Cantelli lemma, $\|\boldsymbol{\lambda}\|<\infty$ a.s., meaning that there are a.s. finitely many levels $y$ where the horizontal direction is randomly perturbed with respect to the direction determined by the periodic function; if $\beta<1$, then $\|\boldsymbol{\lambda}\|=\infty$ a.s.

An extreme choice of "random" perturbation is when $\boldsymbol{\lambda}$ is a deterministic $\{0,1\}$-valued sequence. We have then the following

Proposition 1.8. When $\boldsymbol{\lambda}$ is a deterministic $\{0,1\}$-valued sequence with $\|\boldsymbol{\lambda}\|<\infty$, then the simple random walk is recurrent.

Note however that the previous proposition does not provide us with a necessary condition for recurrence. We shall give in $\$ 5$ the following

Counter-example 1.9. There are deterministic $\{0,1\}$-sequences $\boldsymbol{\lambda}$, with $\|\boldsymbol{\lambda}\|=$ $\infty$ (infinitely many deterministic defects), leading nevertheless to recurrent random walks. 


\section{Technical preliminaries}

Since the general framework developed in [3] is still useful, we only recall here the basic facts. It is always possible to choose a sufficiently large abstract probability space $(\Omega, \mathscr{A}, \mathbb{P})$ on which are defined all the sequences of random variables we shall use, namely $\left(\rho_{y}\right),\left(\lambda_{y}\right)$, etc. and in particular the Markov chain $\left(\mathbf{M}_{n}\right)_{n \in \mathbb{N}}$ itself. When the initial probability of the chain is $v$, then obviously $\mathbb{P}:=\mathbb{P}_{v}$. When $v=\delta_{x}$ we write simply $\mathbb{P}_{x}$ instead of $\mathbb{P}_{\delta_{x}}$.

The idea of the proof is to decompose the stochastic process $\left(\mathbf{M}_{n}\right)_{n \in \mathbb{N}}$ into a vertical skeleton - obtained by the vertical projection of $\left(\mathbf{M}_{n}\right)$ that is stripped out of the waiting times corresponding to the horizontal moves - and a horizontal component. More precisely, define $T_{0}:=0$ and for $k \geq 1$ recursively: $T_{k}=\inf \left\{n>T_{k-1}:\left\langle\mathbf{M}_{n}-\mathbf{M}_{n-1} \mid \mathbf{e}_{2}\right\rangle \neq 0\right\}$. Introduce then the sequences $\psi_{k}=$ $\left\langle\mathbf{M}_{T_{k}}-\mathbf{M}_{T_{k-1}} \mid \mathbf{e}_{2}\right\rangle$ for $k \geq 1$, and $Y_{n}=\sum_{k=1}^{n} \psi_{k}$, for $n \geq 0$ (with the convention $\left.Y_{0}=0\right)$. The process $\left(Y_{n}\right)$ is a simple random walk on the vertical axis, called the vertical skeleton; its occupation measure of level $\{y\}$ is denoted $\eta_{n}(y)=$ $\sum_{k=0}^{n} \mathbb{1}_{\{y\}}\left(Y_{k}\right)$. Similarly, we define the sequences of waiting times. For all $y \in \mathbb{Z}$ define $S_{0}(y):=-1$ and recursively for $k \geq 1: S_{k}(y)=\inf \left\{l>S_{k-1}(y): Y_{l}=y\right\}$. The random variables $\xi_{k}^{(y)}=T_{S_{k}(y)+1}-T_{S_{k}(y)}-1$ represent then the waiting time at level $y$ during the $k^{\text {th }}$ visit at that level. Due to strong Markov property, the doubly infinite sequence $\left(\xi_{k}^{(y)}\right)_{y \in \mathbb{Z}, k \in \mathbb{N}^{*}}$ are independent $\mathbb{N}$-valued random variables with geometric distribution of parameter $p=1 / 3 ; q$ always stands for $1-p$ in the sequel.

Definition 2.1. Suppose the vertical skeleton and the environments of the orientations are given. Let $\left(\xi_{n}^{(y)}\right)_{n \in \mathbb{N}, y \in \mathbb{Z}}$ be the previously defined doubly infinite sequence of geometric random variables of parameter $p=1 / 3$ and $\eta_{n}(y)$ the occupation measures of the vertical skeleton. We call horizontally embedded random walk the process $\left(X_{n}\right)_{n \in \mathbb{N}}$ with

$$
X_{n}=\sum_{y \in \mathbb{Z}} \varepsilon_{y} \sum_{i=1}^{\eta_{n-1}(y)} \xi_{i}^{(y)}, n \in \mathbb{N} .
$$

Lemma 2.2. (See [3, lemma 2.7]). Let $T_{n}=n+\sum_{y \in \mathbb{Z}} \sum_{i=1}^{\eta_{n-1}(y)} \xi_{i}^{(y)}$ be the instant just after the random walk $\left(\mathbf{M}_{k}\right)$ has performed its $n^{\text {th }}$ vertical move (with the convention that the sum $\sum_{i}$ vanishes whenever $\left.\eta_{n-1}(y)=0\right)$. Then $\mathbf{M}_{T_{n}}=\left(X_{n}, Y_{n}\right)$.

Define $\sigma_{0}=0$ and recursively, for $n=1,2, \ldots, \sigma_{n}=\inf \left\{k>\sigma_{n-1}: Y_{k}=0\right\}>$ $\sigma_{n-1}$, the $n^{\text {th }}$ return to the origin for the vertical skeleton. Then obviously, $\mathbf{M}_{T_{\sigma_{n}}}=$ $\left(X_{\sigma_{n}}, 0\right)$. To study the recurrence or the transience of $\left(\mathbf{M}_{k}\right)$, we must study how often $\mathbf{M}_{k}=(0,0)$. Now, $\mathbf{M}_{T_{k}}=(0,0)$ if and only if $X_{k}=0$ and $Y_{k}=0$. Since $\left(Y_{k}\right)$ is a simple random walk, the event $\left\{Y_{k}=0\right\}$ is realised only at the instants $\sigma_{n}$, $n=0,1,2, \ldots$. 
Remark 2.3. The significance of the random variable $X_{n}$ is the horizontal displacement after $n$ vertical moves of the skeleton $\left(Y_{l}\right)$. Notice that the random walk $\left(X_{n}\right)$ has unbounded (although integrable) increments. As a matter of fact, they are signed integer-valued geometric random variables. Contrary to $\left(X_{n}\right)$, the increments of the process $\left(X_{\sigma_{n}}\right)_{n \in \mathbb{N}}$, sampled at instants $\sigma_{n}$, are unbounded with heavy-tails.

Recall that all random variables are defined on the same probability space $(\Omega, \mathscr{A}, \mathbb{P})$; introduce the following sub- $\sigma$-algebras: $\mathscr{H}=\sigma\left(\xi_{i}^{(y)} ; i \in \mathbb{N}^{*}, y \in \mathbb{Z}\right), \mathscr{G}=$ $\sigma\left(\rho_{y}, \lambda_{y} ; y \in \mathbb{Z}\right)$, and $\mathscr{F}_{n}=\sigma\left(\psi_{i} ; i=1, \ldots, n\right)$, with $\mathscr{F}_{\mathscr{F}_{\infty}}$.

Lemma 2.4. (See [3, lemma 2.8])

$$
\sum_{l=0}^{\infty} \mathbb{P}\left(\mathbf{M}_{l}=(0,0) \mid \mathscr{F} \vee \mathscr{G}\right)=\sum_{n=0}^{\infty} \mathbb{P}\left(I\left(X_{\sigma_{n}}, \varepsilon_{0} \xi_{0}^{0}\right) \ni 0 \mid \mathscr{F} \vee \mathscr{G}\right)
$$

where, $\xi_{0}^{0}$ has the same law as $\xi_{1}^{(0)}$ and, for $x \in \mathbb{Z}, z \in \mathbb{N}$, and $\varepsilon= \pm 1, I(x, \varepsilon z)=$ $\{x, \ldots, x+z\}$ if $\varepsilon=+1$ and $\{x-z, \ldots, x\}$ if $\varepsilon=-1$.

Lemma 2.5. (See [3, lemma 2.9])

1. If $\sum_{n=0}^{\infty} \mathbb{P}_{0}\left(X_{\sigma_{n}}=0 \mid \mathscr{F} \vee \mathscr{G}\right)=\infty$ then $\sum_{l=0}^{\infty} \mathbb{P}\left(\mathbf{M}_{l}=(0,0) \mid \mathscr{F} \vee \mathscr{G}\right)=\infty$.

2. If $\left(X_{\sigma_{n}}\right)_{n \in \mathbb{N}}$ is transient then $\left(M_{n}\right)_{n \in \mathbb{N}}$ is also transient.

Let $\xi$ be a geometric random variable equidistributed with $\xi_{i}^{(y)}$. Denote

$$
\chi(\theta)=\mathbb{E} \exp (i \theta \xi)=\frac{q}{1-p \exp (i \theta)}=r(\theta) \exp (i \alpha(\theta)), \quad \theta \in[-\pi, \pi]
$$

its characteristic function, where

$$
r(\theta)=|\chi(\theta)|=\frac{q}{\sqrt{q^{2}+2 p(1-\cos \theta)}}=r(-\theta) ; \alpha(\theta)=\arctan \frac{p \sin \theta}{1-p \cos \theta}=-\alpha(-\theta) .
$$

Notice that $r(\theta)<1$ for $\theta \in[-\pi, \pi] \backslash\{0\}$. Then

$$
\begin{aligned}
\mathbb{E} \exp \left(i \theta X_{n}\right) & =\mathbb{E}\left(\mathbb{E}\left(\exp \left(i \theta X_{n}\right) \mid \mathscr{F} \vee \mathscr{G}\right)\right)=\mathbb{E}\left(\mathbb{E}\left(\exp \left(i \theta \sum_{y \in \mathbb{Z}} \varepsilon_{y} \sum_{i=1}^{\eta_{n-1}(y)} \xi_{i}^{(y)} \mid \mathscr{F} \vee \mathscr{G}\right)\right)\right. \\
& =\mathbb{E}\left(\prod_{y \in \mathbb{Z}} \chi\left(\theta \varepsilon_{y}\right)^{\eta_{n-1}(y)}\right) .
\end{aligned}
$$




\section{Proof of transience}

Introduce, as was the case in [3], constants $\delta_{i}>0$ for $i=1,2,3$ and for $n \in \mathbb{N}$ the sequence of events $A_{n}=A_{n, 1} \cap A_{n, 2}$ and $B_{n}$ defined by

$$
\begin{gathered}
A_{n, 1}=\left\{\omega \in \Omega: \max _{0 \leq k \leq 2 n}\left|Y_{k}\right|<n^{\frac{1}{2}+\delta_{1}}\right\} ; A_{n, 2}=\left\{\omega \in \Omega: \max _{y \in \mathbb{Z}} \eta_{2 n-1}(y)<n^{\frac{1}{2}+\delta_{2}}\right\}, \\
B_{n}=\left\{\omega \in A_{n}:\left|\sum_{y \in \mathbb{Z}} \varepsilon_{y} \eta_{2 n-1}(y)\right|>n^{\frac{1}{2}+\delta_{3}}\right\} ;
\end{gathered}
$$

the range of possible values for $\delta_{i}, i=1,2,3$, will be chosen later (see the end of the proof of proposition 3.4). Obviously $A_{n, 1}, A_{n, 2}$ and hence $A_{n}$ belong to $\mathscr{F}_{2 n}$; moreover $B_{n} \subseteq A_{n}$ and $B_{n} \in \mathscr{F}_{2 n} \vee \mathscr{G}$. We denote in the sequel generically $d_{n, i}=n^{\frac{1}{2}+\delta_{i}}$, for $i=1,2,3$.


as $1=\mathbb{1}_{B_{n}}+\mathbb{1}_{A_{n} \backslash B_{n}}+\mathbb{1}_{A_{n}^{c}}$, we get $p_{n}=p_{n, 1}+p_{n, 2}+p_{n, 3}$, where $p_{n}=\mathbb{P}\left(X_{2 n}=\right.$ $\left.0 ; Y_{2 n}=0\right), p_{n, 1}=\mathbb{P}\left(X_{2 n}=0 ; Y_{2 n}=0 ; B_{n}\right), p_{n, 2}=\mathbb{P}\left(X_{2 n}=0 ; Y_{2 n}=0 ; A_{n} \backslash B_{n}\right)$, and $p_{n, 3}=\mathbb{P}\left(X_{2 n}=0 ; Y_{2 n}=0 ; A_{n}^{c}\right)$. By repeating verbatim the reasoning of propositions 4.1 and 4.3 of [3], we get

Proposition 3.1. For large $n$, there exist $\delta>0$ and $\delta^{\prime}>0$ and $c>0$ and $c^{\prime}>0$ such that

$$
p_{n, 1}=\mathscr{O}\left(\exp \left(-c n^{\delta}\right)\right) \text { and } p_{n, 3}=\mathscr{O}\left(\exp \left(-c^{\prime} n^{\delta^{\prime}}\right)\right)
$$

Consequently $\sum_{n \in \mathbb{N}}\left(p_{n, 1}+p_{n, 3}\right)<\infty$. The proof will be complete if we show that $\sum_{n \in \mathbb{N}} p_{n, 2}<\infty$.

Lemma 3.2. On the set $A_{n} \backslash B_{n}$, we have - uniformly on $\mathscr{F} \vee \mathscr{G}-$

$$
\mathbb{P}\left(X_{2 n}=0 \mid \mathscr{F} \vee \mathscr{G}\right)=\mathscr{O}\left(\sqrt{\frac{\ln n}{n}}\right) .
$$

Proof. Use the $\mathscr{F} \vee \mathscr{G}$-measurability of the variables $\left(\varepsilon_{y}\right)_{y \in \mathbb{Z}}$ and $\left(\eta_{n}(y)\right)_{y \in \mathbb{Z}, n \in \mathbb{N}}$ to express the conditional characteristic function of the variable $X_{2 n}$ as follows:

$$
\chi_{1}(\theta)=\mathbb{E}\left(\exp \left(i \theta X_{2 n}\right) \mid \mathscr{F} \vee \mathscr{G}\right)=\prod_{y \in \mathbb{Z}} \chi\left(\theta \varepsilon_{y}\right)^{\eta_{2 n-1}(y)} .
$$

Hence, $\mathbb{P}\left(X_{2 n}=0 \mid \mathscr{F} \vee \mathscr{G}\right)=\frac{1}{2 \pi} \int_{-\pi}^{\pi} \chi_{1}(\theta) d \theta$. Now use the decomposition of $\chi$ into a the modulus part, $r(\theta)$ - that is an even function of $\theta$ - and the angular part of $\alpha(\theta)$ and the fact that there is a constant $K<1$ such that for $\theta \in[-\pi,-\pi / 2] \cup$ $[\pi / 2, \pi]$ we can bound $r(\theta)<K$ to majorise

$$
\mathbb{P}\left(X_{2 n}=0 \mid \mathscr{F} \vee \mathscr{G}\right) \leq \frac{1}{\pi} \int_{0}^{\pi / 2} r(\theta)^{2 n} d \theta+\mathscr{O}\left(K^{n}\right)
$$


Fix $a_{n}=\sqrt{\frac{\ln n}{n}}$ and split the integration integral [0, $\left.\pi / 2\right]$ into [0, $\left.a_{n}\right] \cup\left[a_{n}, \pi / 2\right]$. For the first part, we majorise the integrand by 1 , so that $\int_{0}^{a_{n}} r(\theta)^{2 n} d \theta \leq a_{n}$.

For the second part, we use the fact that $r(\theta)$ is decreasing for $\theta \in[0, \pi / 2]$. Hence $\frac{1}{\pi} \int_{a_{n}}^{\pi / 2} r(\theta)^{2 n} d \theta \leq \frac{1}{2} r\left(a_{n}\right)^{2 n}$. Now, $\lim _{n \rightarrow \infty} a_{n}=0$, hence for large $n$ it is enough to study the behaviour of $r$ near 0 , namely $r(\theta)=1-\frac{3}{8} \theta^{2}+\mathscr{O}\left(\theta^{4}\right)$. It follows that $r\left(a_{n}\right)^{2 n}=\left(1-\frac{3}{4} \frac{\ln n}{2 n}\right)^{2 n}=\exp \left(-\frac{3}{4} \ln n\right)=n^{-\frac{3}{4}}$. Since the estimate of the first part dominates, the result follows.

Lemma 3.3. Let d be a positive integer, $Z$ an integer-valued random variable with law $\mu_{Z}$, and $G$ a centred Gaussian random variable with variance $d^{2}$, but otherwise independent of $Z$. Then, there exists a constant $C>0$ (independent of $d$ and of the law of $Z$ ) such that

$$
\mathbb{P}\left(|Z| \leq \frac{d}{2}\right) \leq C \mathbb{P}(|Z+G| \leq d) .
$$

Proof. Denote by $\gamma(g)=\frac{1}{\sqrt{2 \pi d^{2}}} \exp \left(-\frac{g^{2}}{2 d^{2}}\right)$ the density of the Gaussian random variable $G$ and observe that on $\left[-\frac{d}{2}, \frac{d}{2}\right]$ the density is minorised by $\gamma(g) \geq \frac{2 C^{-1}}{d}$ with $C=\sqrt{2 \pi e}$. Then

$$
\mathbb{P}(|Z+G| \leq d) \geq \int_{-\frac{d}{2}}^{\frac{d}{2}} \mu_{Z}([-d-g, \ldots, d-g]) \gamma(g) d g \geq 2 C^{-1} \mu_{Z}\left(\left\{-\frac{d}{2}, \ldots, \frac{d}{2}\right\}\right) .
$$

Proposition 3.4. For all $\beta<1$, there exists $a \delta_{\beta}>0$ such that - uniformly in $\mathscr{F}$ - for all large $n$

$$
\mathbb{P}\left(A_{n} \backslash B_{n} \mid \mathscr{F}\right)=\mathscr{O}\left(n^{-\delta_{\beta}}\right) .
$$

Proof. The required probability is an estimate, on the event $A_{n}$, of the conditional probability $\mathbb{P}\left(\left|\sum_{y \in \mathbb{Z}} \zeta_{y, n}\right| \leq d_{n, 3} \mid \mathscr{F}\right)$, where we denote $\zeta_{y, n}=\varepsilon_{y} \eta_{2 n-1}(y)$. Extend the probability space $(\Omega, \mathscr{A}, \mathbb{P})$ to carry an auxilliary variable $G$ assumed to be centred Gaussian with variance $d_{n, 3}^{2}$, (conditionally on $\mathscr{F}$ ) independent of the $\zeta_{y, n}$ 's. Since $G$ is a symmetric random variable and $\left[-d_{n, 3}, d_{n, 3}\right]$ is a symmetric set around 0 , then by lemma 3.3, there exists a positive constant $c:=\sqrt{2 \pi e}$ (hence independent of $n$ ) such that

$$
\mathbb{P}\left(\left|\sum_{y \in \mathbb{Z}} \zeta_{y, n}\right| \leq d_{n, 3} \mid \mathscr{F}\right) \leq c \mathbb{P}\left(\left|\sum_{y \in \mathbb{Z}} \zeta_{y, n}+G\right| \leq d_{n, 3} \mid \mathscr{F}\right) .
$$

Let $\chi_{2}(t)=\mathbb{E}\left(\exp \left(i t \sum_{y} \zeta_{y, n}\right) \mid \mathscr{F}\right)=\prod_{y} A_{y, n}(t)$, where $A_{y, n}(t)=\mathbb{E}\left(\exp \left(i t \zeta_{y, n} \mid \mathscr{F}\right)\right.$, and $\chi_{3}(t)=\mathbb{E}(\exp (i t G) \mid \mathscr{F})=\exp \left(-t^{2} d_{n, 3}^{2} / 2\right)$. Therefore, $\mathbb{E}\left(\exp \left(i t\left(\sum_{y} \zeta_{y, n}+G\right)\right) \mid \mathscr{F}\right)=$ $\chi_{2}(t) \chi_{3}(t)$, and using the Plancherel's formula,

$$
\mathbb{P}\left(\left|\sum_{y \in \mathbb{Z}} \zeta_{y, n}+G\right| \leq d_{n, 3} \mid \mathscr{F}\right)=\frac{d_{n, 3}}{\pi} \int_{\mathbb{R}} \frac{\sin \left(t d_{n, 3}\right)}{t d_{n, 3}} \chi_{2}(t) \chi_{3}(t) d t \leq C d_{n, 3} I,
$$


where $I=\int_{\mathbb{R}}\left|\chi_{2}(t)\right| \exp \left(-t^{2} d_{n, 3}^{2} / 2\right) d t$. Fix $b_{n}=\frac{n^{\delta_{4}}}{d_{n, 3}}$, for some $\delta_{4}>0$ and split the integral defining $I$ into $I_{1}+I_{2}$, the first part being for $|t| \leq b_{n}$ and the second for $|t|>b_{n}$.

We have

$$
\begin{aligned}
I_{2} & \leq C \int_{|t|>b_{n}} \exp \left(-t^{2} d_{n, 3}^{2} / 2\right) \frac{d t}{2 \pi}=\frac{C}{d_{n, 3}} \int_{|s|>n^{\delta_{4}}} \exp \left(-s^{2} / 2\right) \frac{d s}{2 \pi} \\
& \leq 2 \frac{C}{d_{n, 3}} \frac{1}{n^{\delta_{4}}} \frac{\exp \left(-n^{\left.2 \delta_{4} / 2\right)}\right.}{2 \pi},
\end{aligned}
$$

because the probability that a centred normal random variable of variance 1 , whose density is denoted $\phi$, exceeds a threshold $x>0$ is majorised by $\frac{\phi(x)}{x}$.

For $I_{1}$ we get, $I_{1} \leq \int_{|t| \leq b_{n}} \prod_{y}\left|A_{y, n}(t)\right| d t$.

Assume for the moment that the inequality $\left|t \eta_{2 n-1}(y)\right| \leq 1$ holds. Use then the fact that $\cos (x) \leq 1-\frac{x^{2}}{4}$, valid for $|x| \leq 1$, to write

$$
\begin{aligned}
\left|A_{y, n}(t)\right| & =\left|\mathbb{E}_{0}\left(\exp \left(i t \varepsilon_{y} \eta_{2 n-1}(y)\right) \mathscr{F}\right)\right| \\
& =\left|\left(1-\frac{c}{|y|^{\beta}}\right) \exp \left(i t \eta_{2 n-1}(y) f(y)\right)+\frac{c}{|y|^{\beta}} \cos \left(t \eta_{2 n-1}(y)\right)\right| \\
& \leq 1-\frac{c}{|y|^{\beta}}+\frac{c}{|y|^{\beta}}\left(1-\frac{t^{2} \eta_{2 n-1}^{2}(y)}{4}\right) \\
& =1-\frac{c t^{2} \eta_{2 n-1}^{2}(y)}{4|y|^{\beta}} \leq \exp \left(-\frac{c t^{2} \eta_{2 n-1}^{2}(y)}{4|y|^{\beta}}\right) .
\end{aligned}
$$

The assumed inequality $\left|t \eta_{2 n-1}(y)\right| \leq 1$ is verified whenever the constants $\delta_{2}, \delta_{3}$ and $\delta_{4}$ are chosen so that $\delta_{2}+\delta_{4}-\delta_{3}<0$ holds, because $|t| \leq b_{n}$ and $b_{n}=\frac{n^{d_{4}}}{n^{1 / 2+\delta_{3}}}$, whereas $\eta_{2 n-1}(y) \leq n^{1 / 2+\delta_{2}}$. Therefore,

$$
\left|\chi_{2}(t)\right| \leq \prod_{y} \exp \left(-\frac{t^{2}}{4} \eta_{2 n-1}^{2}(y) \frac{c}{|y|^{\beta}}\right) .
$$

Now, define $\pi_{n}(y)=\frac{\eta_{2 n-1}(y)}{2 n}$; obviously $\sum_{y} \pi_{n}(y)=1$, establishing that $\left(\pi_{n}(y)\right)_{y}$ is a probability measure on $\mathbb{Z}$. Therefore, applying Hölder's inequality we obtain $I_{1} \leq \prod_{y}^{\prime} J_{n}(y)^{\pi_{n}(y)}$, where $\prod_{y}^{\prime}$ means that the product runs over those $y$ such that $\eta_{2 n-1}(y) \neq 0$ and

$$
\begin{aligned}
& J_{n}(y)=\int_{-b_{n}}^{b_{n}} \exp \left(-\frac{t^{2}}{4} \eta_{2 n-1}^{2}(y) \frac{c}{|y|^{\beta}} \frac{1}{\pi_{n}(y)}\right) d t \\
& =\sqrt{\frac{2 \pi|y|^{\beta}}{c n \eta_{2 n-1}(y)}} \int_{-b_{n} \sqrt{\frac{c n \eta_{2 n-1}(y)}{\left.|y|\right|^{\beta}}}}^{b_{n} \sqrt{\frac{c n \eta_{2 n-1}(y)}{\mid y \beta}}} \exp \left(-v^{2} / 2\right) \frac{d v}{\sqrt{2 \pi}} \\
& \leq \sqrt{\frac{4 \pi}{c}} \exp \left(-\log 2 n-\frac{1}{2} \log \pi_{n}(y)+\frac{\beta}{2} \log |y|\right) \text {. }
\end{aligned}
$$


We conclude that

$$
I_{1} \leq \prod_{y}^{\prime} J_{n}(y)^{\pi_{n}(y)} \leq \sqrt{\frac{2 \pi}{c}} \exp \left(-\log 2 n+\frac{1}{2} H\left(\pi_{n}\right)+\frac{\beta}{2} \sum_{y} \pi_{n}(y) \log |y|\right)
$$

and $H\left(\pi_{n}\right)$ is the entropy of the probability measure $\pi_{n}$, reading (with the convention $0 \log 0=0$ )

$$
H\left(\pi_{n}\right):=-\sum_{y} \pi_{n}(y) \log \pi_{n}(y) \leq \log \operatorname{card} C_{n},
$$

where $C_{n}:=\operatorname{supp} \pi_{n}$ and, on $A_{n}, \operatorname{card} C_{n} \leq 2 n^{\frac{1}{2}+\delta_{1}}$. We conclude that we can always chose the parameters $\delta_{1}$ and $\delta_{3}$ such that, for every $\beta<1$ there exists a parameter $\delta_{\beta}>0$ such that $d_{n, 3} I_{1} \leq C n^{-\delta_{\beta}}$.

\section{Corollary 3.5.}

$$
\sum_{n \in \mathbb{N}} p_{n, 2}<\infty
$$

Proof. Recall that for the standard random walk $\mathbb{P}\left(Y_{2 n}=0\right)=\mathscr{O}\left(n^{-1 / 2}\right)$; combining with the estimates obtained in 3.2 and 3.4, we have

$$
\begin{aligned}
p_{n, 2} & =\mathbb{P}\left(X_{2 n}=0 ; Y_{2 n}=0 ; A_{n} \backslash B_{n}\right) \\
& =\mathbb{E}\left(\mathbb{E}\left(\mathbb{1}_{Y_{2 n}=0}\left[\mathbb{E}\left(\mathbb{1}_{A_{n} \backslash B_{n}} \mathbb{P}\left(X_{2 n}=0 \mid \mathscr{F} \vee \mathscr{G}\right) \mid \mathscr{F}\right)\right]\right)\right) \\
& =\mathscr{O}\left(n^{-1 / 2} n^{-\delta_{\beta}} \sqrt{\frac{\ln n}{n}}\right)=\mathscr{O}\left(n^{-\left(1+\delta_{\beta}\right)} \sqrt{\ln n}\right),
\end{aligned}
$$

proving thus the summability of $p_{n, 2}$.

Proof of the statement on transience of the theorem 1.5: $p_{n}=p_{n, 1}+p_{n, 2}+p_{n, 3}$ is summable because all the partial probabilities $p_{n, i}$, for $i=1,2,3$ are all shown to be summable.

\section{Proof of recurrence}

We define additionally the following sequence of random times:

$$
\tau_{0} \equiv 0 \text { and } \tau_{n+1}=\inf \left\{k: k>\tau_{n},\left|Y_{k}-Y_{\tau_{n}}\right|=Q\right\} \text { for } n \geq 0 .
$$

The random variables $\left(\tau_{n+1}-\tau_{n}\right)_{n \geq 0}$ are independent and for all $n$ the variable $\tau_{n+1}-\tau_{n}$ has the same distribution (under $\mathbb{P}_{0}$ ) as $\tau_{1}$. It is easy to show further (see proposition 1.13.4 of the textbook [1] for instance) that these random variables have exponential moments i.e. $\mathbb{E}_{0}\left(\exp \left(\alpha \tau_{1}\right)\right)<\infty$ for $|\alpha|$ sufficiently small. 
Let $\mathbb{Z}_{Q}=\mathbb{Z} / Q \mathbb{Z}=\{0,1, \ldots, Q-1\}$ with integer addition replaced by addition modulo $Q$ and for any $y \in \mathbb{Z}$ denote by $\bar{y}=y \bmod Q \in \mathbb{Z}_{Q}$. Consistently, we define $\bar{Y}_{n}=Y_{n} \bmod Q$.

Lemma 4.1. Define for $n \geq 1$ and $\bar{y} \in \mathbb{Z}_{Q}$,

$$
N_{n}(\bar{y}):=\bar{\eta}_{\tau_{n-1}, \tau_{n}-1}(\bar{y})=\sum_{k=\tau_{n-1}}^{\tau_{n}-1} \mathbb{1}_{\bar{y}}\left(\bar{Y}_{k}\right) .
$$

Then, for every $\bar{y} \in \mathbb{Z}_{Q}$,

1. the conditional laws of $N_{1}(\bar{y})$ with respect to the events $\left\{Y_{\tau_{1}}=Q\right\}$ and $\left\{Y_{\tau_{1}}=\right.$ $-Q\}$ are the same, and

2. the following equalities hold

$$
\mathbb{E}_{0} N_{1}(\bar{y})=\mathbb{E}_{0}\left(N_{1}(\bar{y}) \mid Y_{\tau_{1}}=Q\right)=\mathbb{E}_{0}\left(N_{1}(\bar{y}) \mid Y_{\tau_{1}}=-Q\right)=\frac{\mathbb{E}_{0} \tau_{1}}{Q} .
$$

Proof. 1. Denote by $U:=\left\{Y_{\tau_{1}}=Q\right\}$ and $D:=\left\{Y_{\tau_{1}}=-Q\right\}$ the sets of conditioning. Assume that $Y$ is a trajectory in $U$ and define $R:=\max \{t: 0 \leq t<$ $\tau_{1}, Y_{t}=0$ \}. Now, between times 0 and $R$, the path $Y$ wanders around the level 0 . For times $t$ such that $R<t<\tau_{1}$, the path remains strictly confined within the (interior of the) strip.

For any trajectory $Y$ in $U$, we shall define a new trajectory $V$ - bijectively determined from $Y$ - belonging to $D$ as follows:

$$
V_{t}= \begin{cases}Y_{t} & \text { for } \quad 0 \leq t \leq R \\ Y_{\tau_{1}-(t-R)}-Q & \text { for } \quad R \leq t \leq \tau_{1}\end{cases}
$$

Obviously, the above construction is a bijection. Hence for trajectories not in $U$ (i.e. trajectories in $D$ ) the modified trajectory is defined inverting the previous transformation. The figure 1 illustrates the construction (modified reflection principle).

On denoting by $\eta$ the occupation measure of the process $Y$ and by $\kappa$ the one of $V$, we have $\bar{\kappa}_{\tau_{1}-1}(\bar{y}):=\sum_{i=0}^{\tau_{1}-1} \mathbb{1}_{\{\bar{y}\}}\left(\bar{V}_{i}\right)=\sum_{i=0}^{\tau_{1}-1} \mathbb{1}_{\{\bar{y}\}}\left(\bar{Y}_{i}\right)=: \bar{\eta}_{\tau_{1}-1}(\bar{y})$ by construction of the path $V$. This remark implies that $\bar{\eta}_{\tau_{1}-1}(\cdot)$ and $\bar{\kappa}_{\tau_{1}-1}(\cdot)$ have the same law.

2. Since the random walk $\left(Y_{n}\right)$ is symmetric, the probability of exiting the strip of width $Q$ by up-crossing is the same as for a down-crossing. Hence $\mathbb{E}_{0} N_{1}(\bar{y})=\frac{1}{2} \mathbb{E}_{0}\left(N_{1}(\bar{y}) \mid Y_{\tau_{1}}=Q\right)+\frac{1}{2} \mathbb{E}_{0}\left(N_{1}(\bar{y}) \mid Y_{\tau_{1}}=-Q\right)$. This remark, combined with the equality of conditional laws established in 1. establishes the leftmost and the central equalities of the statement.

To prove the rightmost equality, let $g: \mathbb{Z} \rightarrow \mathbb{R}$ be a bounded function and denote by $S_{n}[g]=\sum_{k=0}^{n-1} g\left(Y_{k}\right)$. On defining $W_{n}[g]=\sum_{k=\tau_{n}}^{\tau_{n+1}-1} g\left(Y_{k}\right)$ and 

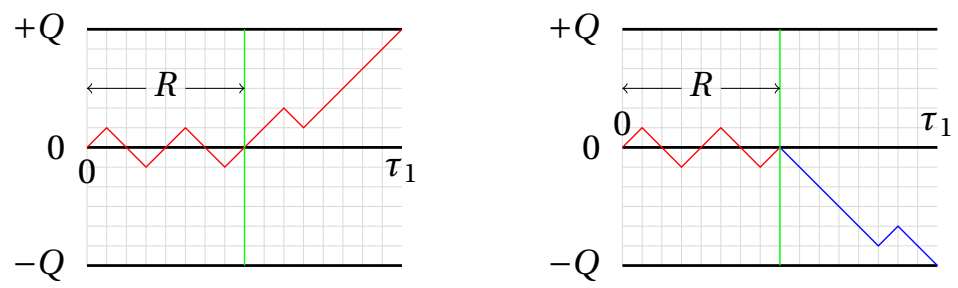

Figure 1: Illustration of the modified reflexion principle. The left figure depicts a detail of the up-crossing excursion, occurring between times 0 and $\tau_{1}$. The left figure depicts the details of a new admissible path bijectively obtained from $Y$ by defining it as identical to $Y$ for the times $0 \leq t \leq R$ and then by reverting the flow of time and displacing the remaining portion of the path by $-Q$, as explained in the text.

$R_{n}=\max \left\{k: \tau_{k} \leq n\right\}$, we have the decomposition:

$$
S_{n}[g]=\sum_{k=0}^{R_{n}} W_{k}[g]-\sum_{k=n}^{\tau_{R_{n+1}-1}} g\left(Y_{k}\right) .
$$

Since $\tau_{R_{n}+1}-n \leq \tau_{R_{n}+1}-\tau_{R_{n}}$, we have, thanks to the boundedness of $g$, that $\frac{1}{n}\left|\sum_{k=n}^{\tau_{R_{n}+1}-1} g\left(Y_{k}\right)\right| \leq \frac{\tau_{R_{n+1}}-\tau_{R_{n}}}{n} \sup _{y \in \mathbb{Z}}|g(z)|$. Since

$$
\begin{aligned}
\mathbb{P}\left(\tau_{R_{n+1}}-\tau_{R_{n}}=l\right) & \leq \sum_{k=0}^{n} \mathbb{P}\left(\tau_{k+1}-\tau_{k}=l ; R_{n}=k\right) \\
& \leq \sum_{k=0}^{n} \mathbb{P}\left(\tau_{k+1}-\tau_{k}=l\right),
\end{aligned}
$$

it follows that for all $\varepsilon>0$, we have $\sum_{k=1}^{n} \mathbb{P}\left(\tau_{k+1}-\tau_{k} \geq \varepsilon n\right) \leq n \mathbb{P}\left(\tau_{1} \geq \varepsilon n\right)$ which tends to 0 , when $n \rightarrow \infty$, thanks to Markov inequality and the existence of exponential moments for $\tau_{1}$.

It remains to estimate $\frac{S_{n}[g]}{n}$ by $\frac{R_{n}}{n} \frac{1}{R_{n}} \sum_{k=0}^{R_{n}} W_{k}[g]$. Obviously $R_{n} \rightarrow \infty$ a.s., as $n \rightarrow \infty$, and, by the renewal theorem (see p. 221 of [1] for instance), $\frac{R_{n}}{n} \rightarrow \frac{1}{\mathbb{E}_{0} \tau_{1}}$ a.s. Fix any $\bar{y} \in \mathbb{Z}_{Q}$ and choose $g(z):=\mathbb{1}_{\{\bar{y}\}}(z \bmod Q)$. For this $g$, we have $S_{n}[g]=\bar{\eta}_{n}(\bar{y})$, where $\bar{\eta}_{n}(\bar{y})=\sum_{k=0}^{n-1} \mathbb{1}_{\{\bar{y}\}}\left(\bar{Y}_{k}\right)$. But $\left(\bar{Y}_{k}\right)$ is a simple random walk on the finite set $\mathbb{Z}_{Q}$ therefore admits a unique invariant probability $\bar{\pi}(\bar{y})=\frac{1}{Q}$. By the ergodic theorem for Markov chains, we have $\frac{S_{n}[g]}{n} \rightarrow \frac{1}{Q}$ a.s.

Additionally, for this choice of $g$, the sequence $\left(W_{k}[g]\right)_{k \in \mathbb{N}}$ are independent random variables, identically distributed as $N_{1}(\bar{y})$. We conclude by applying the law of large numbers to the ratio $\frac{1}{R_{n}} \sum_{k=1}^{R_{n}} W_{k}[g]$. 
To prove almost sure recurrence, it is enough to show $\sum_{k \in \mathbb{N}} \mathbb{P}_{0}\left(X_{\sigma_{k}}=0, Y_{\sigma_{k}}=0 \mid \mathscr{G}\right)=$ $\infty$ a.s. If $\beta>1$ then $\sum_{y} \mathbb{P}\left(\lambda_{y}=1\right)<\infty$; hence, by Borel-Cantelli lemma, there is almost surely a finite number of $y$ 's such that $\lambda_{y}=1$, i.e. the $\mathscr{G}$-measurable random variable $l(\omega)=\max \left\{|y|: \lambda_{y}=1\right\} / Q$ is almost surely finite. Fix an integer $L(\omega) \geq l(\omega)+1$, and introduce the $\mathscr{F} \vee \mathscr{G}$-measurable random sets:

$$
\begin{aligned}
& F_{L, 2 n}(\omega)=\left\{k: 0 \leq k \leq 2 n-1 ;\left|Y_{\tau_{k}(\omega)}(\omega)\right| \leq L(\omega) Q ;\left|Y_{\tau_{k+1}(\omega)}(\omega)\right| \leq L(\omega) Q\right\} \\
& G_{L, 2 n}(\omega)=\left\{k: 0 \leq k \leq 2 n-1 ;\left|Y_{\tau_{k}(\omega)}(\omega)\right| \geq L(\omega) Q ;\left|Y_{\tau_{k+1}(\omega)}(\omega)\right| \geq L(\omega) Q\right\}
\end{aligned}
$$

To simplify notation, we drop explicit reference to the $\omega$ dependence of those sets.

Denote by $\operatorname{Adm}(2 n)$ the set of admissible paths $\mathbf{z}=\left(z_{0}, z_{1}, \ldots, z_{2 n-1}, z_{2 n}\right) \in$ $\mathbb{Z}^{2 n+1}$ satisfying $\left|z_{i+1}-z_{i}\right|=1$ for $i=0, \ldots 2 n-1$ and $z_{0}=0$. For any $\mathbf{z} \in \operatorname{Adm}(2 n)$, we denote $C[\mathbf{z}]$ the cylinder set

$$
C[\mathbf{z}]=\left\{\omega \in \Omega: Y_{0}(\omega)=Q z_{0}=0, Y_{\tau_{1}(\omega)}(\omega)=Q z_{1}, \ldots, Y_{\tau_{2 n}(\omega)}(\omega)=Q z_{2 n}\right\} \in \mathscr{F} .
$$

Denote by $\theta_{k}=X_{\tau_{k+1}}-X_{\tau_{k}}$, for $k \in\{0, \ldots, 2 n-1\}$, and observe that

$$
X_{\tau_{2 n}}=\sum_{k=0}^{2 n-1} \theta_{k}=\sum_{k \in F_{L, 2 n}} \theta_{k}+\sum_{k \in G_{L, 2 n}} \theta_{k}
$$

the sums appearing in the above decomposition referring to disjoint excursions.

Proposition 4.2. For every $\mathbf{z} \in A d m(2 n)$ and every $k \in G_{L, 2 n}(\omega)$, with $\omega \in C[\mathbf{z}]$,

$$
a_{k}:=\mathbb{E}_{0}\left(\theta_{k} \mid C[\mathbf{z}] ; \mathscr{G}\right)=0 .
$$

Proof. Let $\mathbf{z}$ be an arbitrary admissible path and suppose that $k$ corresponds, say, to an up-crossing (i.e. $z_{k+1}-z_{k}=1$ ) and abbreviate to $z:=z_{k}$ and $z+1=$ $z_{k+1}$ in order to simplify notation. Since $\mathbf{z} \in \operatorname{Adm}(2 n)$, then for all $\omega \in C[\mathbf{z}]$, the random times $\tau_{1}, \ldots, \tau_{2 n}$ are compatible with $\mathbf{z}$, meaning, in particular, that

$$
Y_{\tau_{k}}=Q z \text { and } Y_{\tau_{k+1}}=Q z+Q .
$$

The horizontal increments $\left(\theta_{k}\right)_{k \in G_{L, 2 n}}$, conditionally on $C[\mathbf{z}]$ and $\mathscr{G}$, are independent. To simplify notation, introduce the symbol $T:=\tau_{k+1}-\tau_{k}$; obviously, $T \stackrel{\mathrm{d}}{=} \tau_{1}$ conditionally on the starting point; more precisely $\mathbb{P}_{Q z_{k}}(T=t)=\mathbb{P}_{0}\left(\tau_{1}=\right.$ $t$ ), for all $t \in \mathbb{N}$. 
We are now in position to complete the proof of the proposition.

$$
\begin{aligned}
a_{k} & =\mathbb{E}_{0}\left(\sum_{y \in \mathbb{Z}} f(y) \sum_{i=0}^{\eta_{\tau_{k}, \tau_{k+1}-1}(y)} \xi_{i}^{y} \mid C[\mathbf{z}] ; \mathscr{G}\right) \\
& =\mathbb{E}\left(\xi_{0}^{0}\right) \sum_{y=Q z-Q+1}^{Q z+Q-1} f(\bar{y}) \mathbb{E}_{Q z}\left(\eta_{T-1}(y) \mid Y_{T}=Q z+Q ; C[\mathbf{z}] ; \mathscr{G}\right) \mathbb{P}_{Q z}\left(Y_{T}=Q z+Q \mid C[\mathbf{z}] ; \mathscr{G}\right) \\
& =\mathbb{E}\left(\xi_{0}^{0}\right) \sum_{\bar{y} \in \mathbb{Z}_{Q}} f(\bar{y}) \mathbb{E}_{0}\left(N_{1}(\bar{y}) \mid Y_{\tau_{1}}=Q\right) \\
& =\sum_{\bar{y} \in \mathbb{Z}_{Q}} f(\bar{y}) \frac{\mathbb{E}_{0}\left(\tau_{1}\right)}{Q} \mathbb{E}_{\left(\xi_{0}^{0}\right)} \\
& =0,
\end{aligned}
$$

where we used strong Markov property, lemma 4.1, and the centring condition $\sum \bar{y} \in \mathbb{Z}_{Q} f(\bar{y})=0$ to conclude.

The sampled process $Z_{k}=\frac{Y_{\tau_{k}}}{Q} \in \mathbb{Z}$ is a standard simple symmetric nearest neighbour random walk on $\mathbb{Z}$. For $z \in \mathbb{Z}$, define the occupation measure $\oplus_{n}(z):=$ $\varpi(\{z\})=\sum_{k=1}^{n} \mathbb{1}_{\{z\}}\left(Z_{k}\right)$.

Lemma 4.3. Fix $K>0$. For every $\delta>0$ there exists a constant $c>0$ such that for all $n$ sufficiently large,

$$
\mathfrak{P}_{n}=\mathbb{P}_{0}\left(\max _{z:|z| \leq K} \oplus_{2 n}(z)>c \sqrt{n} \mid Z_{2 n}=0\right)<\delta .
$$

Remark 4.4. This lemma will be used in the course of the proof by fixing a $\mathscr{G}$ measurable almost surely finite $K$, while $n$ will tend to infinity. Of course $c=$ $c(K, \delta)$ depends on the choice of $K$ and $\delta$.

Proof. Denote by $m_{n}=\lfloor c \sqrt{n}\rfloor$. Then, by conditional Markov inequality,

$$
\mathfrak{P}_{n} \leq \sum_{z=-K}^{K} \mathbb{P}_{0}\left(\varrho_{2 n}(z)>m_{n} \mid Z_{2 n}=0\right) \leq \sum_{z=-K}^{K} \frac{\mathbb{E}_{0}\left(\varrho_{2 n}(z) \mid Z_{2 n}=0\right)}{m_{n}} .
$$

Now

$$
\begin{aligned}
\mathbb{E}_{0}\left(\varrho_{2 n}(z) \mid Z_{2 n}=0\right) & =\sum_{k=1}^{2 n} \mathbb{P}_{0}\left(Z_{k}=z \mid Z_{2 n}=0\right) \\
& =\sum_{k=1}^{2 n} \frac{P^{k}(0, z) P^{2 n-k}(z, 0)}{P^{2 n}(0,0)}
\end{aligned}
$$

where $P^{l}(0, z)=\mathbb{P}_{0}\left(Z_{l}=z\right)$. For all $z \geq 0$ and all $l \geq z, P^{l}(0, z)=2^{-l}\left(\begin{array}{c}l+z \\ \frac{l}{2}\end{array}\right)$ if $l+z$ is even and 0 otherwise. We majorise $\left(\begin{array}{c}l \\ \frac{l+z}{2}\end{array}\right) \leq\left(\begin{array}{c}l \\ \frac{l}{2}\end{array}\right)$, when $l$ is even and $\left(\begin{array}{c}l \\ \frac{l+z}{2}\end{array}\right) \leq\left(\begin{array}{c}l \\ \frac{l-1}{2}\end{array}\right)$ 
when $l$ is odd. Using Stirling's formula, we see that for all $l$ sufficiently large, the probability $P^{l}(0, z)$ is majorised independently of the parity of $l$ by a term equivalent (for large $l$ ) to $\frac{1}{\sqrt{l}}$. Consquently by choosing an appropriate constant $C$, the same majorisation holds for the remaining finite set of values of $l$. By approximating the sum by an integral, we get finally that

$$
\mathbb{E}_{0}\left(\varrho(z) \mid Z_{2 n}=0\right) \leq C \int_{0}^{2 n} \sqrt{\frac{2 n}{t(2 n-t)}} d t \leq e \sqrt{n} .
$$

We conclude that $\mathfrak{P}_{n} \leq \delta$ provided that $c>\frac{2 K+1}{C}$.

\section{Proof of the recurrence statement of theorem 1.5:}

We shall now fix $K=L$. For $\delta \in] 0,1$ [, let $c=c(K, \delta)$ be as in the previous lemma 4.3. From this very same lemma, we have $\mathbb{P}_{0}\left(\operatorname{card} F_{L, 2 n} \leq c \sqrt{n}\right) \geq 1-\delta$ on the set $\left\{Z_{2 n}=0\right\}$. Fix some constant $d$ and define

ConsAdm $(L, 2 n, d)=\left\{\mathbf{z} \in \operatorname{Adm}(2 n): z_{2 n}=0 ;\left|\left\{k: 0 \leq k<2 n,\left|z_{k}\right| \leq L ;\left|z_{k+1}\right| \leq L\right\}\right| \leq d \sqrt{n}\right\}$

the set of constrained admissible paths. (Here and in the sequel, we use indistinguishably the symbols $|A|$ or $\operatorname{card} A$ to denote the cardinality of the discrete set $A$ ). On the set $\left\{Z_{2 n}=0\right\}$, obviously the equality $\left\{\operatorname{card} F_{L, 2 n} \leq d \sqrt{n}\right\}=$ $\cup_{\mathbf{z} \in \operatorname{Cons} A d m(L, 2 n, d)} C[\mathbf{z}]$ holds.

$$
\begin{aligned}
\mathbb{P}_{0}\left(X_{\tau_{2 n}}=0 ; Y_{\tau_{2 n}}=0 \mid \mathscr{G}\right) & =\mathbb{P}_{0}\left(X_{\tau_{2 n}}=0 ; Z_{2 n}=0 \mid \mathscr{G}\right) \\
& \geq \mathbb{P}_{0}\left(X_{\tau_{2 n}}=0 ; Y_{\tau_{2 n}}=0 ;\left|F_{L, 2 n}\right| \leq d \sqrt{n} \mid\left\{Z_{2 n}=0\right\} ; \mathscr{G}\right) \mathbb{P}_{0}\left(Z_{2 n}=0\right) \\
& =\sum_{\mathbf{z} \in \operatorname{ConsAdm}(L, 2 n, d)} \mathbb{P}_{0}\left(\left\{X_{\tau_{2 n}}=0\right\} \cap C[\mathbf{z}] \mid\left\{Z_{2 n}=0\right\} ; \mathscr{G}\right) \mathbb{P}_{0}\left(Z_{2 n}=0\right) \\
& =\sum_{\mathbf{z} \in \operatorname{ConsAdm}(L, 2 n, d)} \mathbb{P}_{0}\left(X_{\tau_{2 n}}=0 \mid C[\mathbf{z}] ; \mathscr{G}\right) \mathbb{P}_{0}(C[\mathbf{z}] \mid \mathscr{G}) .
\end{aligned}
$$

Now, for any $\mathbf{z} \in$ ConsAdm $(L, 2 n, d)$,

$$
\begin{aligned}
\mathbb{P}_{0}\left(X_{\tau_{2 n}}=0 \mid \mathscr{G}, C[\mathbf{z}]\right) & \geq \sum_{|m| \leq d \sqrt{n}} \mathbb{P}_{0}\left(\sum_{k \in F_{L, 2 n}} \theta_{k}=m ; \sum_{k \in G_{L, 2 n}} \theta_{k}=-m \mid \mathscr{G}, C[\mathbf{z}]\right) \\
& =\sum_{|m| \leq d \sqrt{n}} \mathbb{P}_{0}\left(\sum_{k \in F_{L, 2 n}} \theta_{k}=m \mid \mathscr{G}, C[\mathbf{z}]\right) \mathbb{P}_{0}\left(\sum_{k \in G_{L, 2 n}} \theta_{k}=-m \mid \mathscr{G}, C[\mathbf{z}]\right) .
\end{aligned}
$$

The joint probability factors into the terms appearing in the last line because the $\mathscr{G}$-measurable set-valued random variables $G_{L, 2 n}$ and $F_{L, 2 n}$ take disjoint values, hence the terms in $F_{L, 2 n}$ and $G_{L, 2 n}$ refer to different excursions of the random walk $Y$. Independence follows as a consequence of the strong Markov property.

By the proposition 4.2 , we have $\mathbb{E}\left(\theta_{k} \mid C[\mathbf{z}], \mathscr{G}\right)=0$. The variables $\left(\theta_{k}\right)_{k \in G_{L, 2 n}}$ are independent and identically distributed conditionally to $\mathscr{G}$ and $C[\mathbf{z}]$; their 
common variance, $\sigma^{2}$, is finite because,

$$
\begin{aligned}
\sigma^{2} & =\mathbb{E}_{0}\left(\theta_{k}^{2} \mid \mathscr{G}, C[\mathbf{z}]\right)=\mathbb{E}_{Q z_{k}}\left(\left[\left.\sum_{y} \varepsilon_{y} \sum_{i=0}^{\eta_{\tau_{k}, \tau_{k+1}{ }^{-1}}(y)} \xi_{i}^{y}\right|^{2} \mid \mathscr{G}\right)\right. \\
& \leq \mathbb{E}_{0}\left(\tau_{1}\right) \mathbb{E}\left(\left(\xi_{0}^{0}\right)^{2}\right)+\mathbb{E}_{0}\left(\tau_{1}^{2}\right)\left[\mathbb{E}\left(\xi_{0}^{0}\right)\right]^{2}+\left[\mathbb{E}_{0}\left(\tau_{1}\right)\right]^{2}\left[\mathbb{E}\left(\xi_{0}^{0}\right)\right]^{2}<\infty,
\end{aligned}
$$

where we have used strong Markov property to bound the last term of the first line in the previous formula by the second line.

For $\mathbf{z} \in$ ConsAdm $(L, 2 n, d)$, we have further - on $C[\mathbf{z}]-$ that $2 n-d \sqrt{n} \leq$ $\left|G_{L, 2 n}\right| \leq 2 n$. Hence, for $|m| \leq d \sqrt{n}$, we can apply local limit theorem (see proposition 52.12, p. 706 of [18] for instance), reading

$$
\mathbb{P}_{0}\left(\sum_{k \in G_{L, 2 n}} \theta_{k}=-m \mid \mathscr{G}, C[\mathbf{z}]\right) \geq \frac{c_{1}}{\sqrt{\left|G_{L, 2 n}\right| \sigma^{2}}} \exp \left(-\frac{m^{2}}{2\left|G_{L, 2 n}\right| \sigma^{2}}\right),
$$

to obtain $\mathbb{P}_{0}\left(\sum_{k \in G_{L, 2 n}} \theta_{k}=-m \mid \mathscr{G}, C[\mathbf{z}]\right) \geq \frac{c_{2}}{\sqrt{n}}$, uniformly in $\mathbf{z}$. We can summarise the estimate obtained so far

$\mathbb{P}_{0}\left(X_{\tau_{2 n}}=0, Y_{\tau_{2 n}}=0 \mid \mathscr{G}\right) \geq \frac{c_{3}}{\sqrt{n}} \sum_{\mathbf{z} \in \operatorname{ConsAdm}(L, 2 n, d)} \mathbb{P}_{0}(C[\mathbf{z}] \mid \mathscr{G}) \mathbb{P}_{0}\left(\left|\sum_{k \in F_{L, 2 n}} \theta_{k}\right| \leq d \sqrt{n} \mid C[\mathbf{z}] ; \mathscr{G}\right)$.

Now, $\left\{\left|\sum_{k \in F_{L, 2 n}} \theta_{k}\right| \leq d \sqrt{n}\right\} \supseteq\left\{\sum_{k \in F_{L, 2 n}}\left|\theta_{k}\right| \leq d \sqrt{n}\right\} \supseteq\left\{\sum_{k \in F_{L, 2 n}} \Theta_{k} \leq d \sqrt{n}\right\}$, where $\Theta_{k}=\sum_{y} \sum_{i=\eta_{\tau_{k}(y)}}^{\eta_{\tau_{k+1}}(y)} \xi_{i}^{y}$ are i.i.d. conditionally on $C[\mathbf{z}]$, with finite mean $0 \leq \mu=$ $\mathbb{E} \Theta_{k}=\mathbb{E}\left(\xi_{0}^{0}\right) \mathbb{E}\left(T_{k}\right)<\infty$ and variance $0 \leq \sigma^{2}=\operatorname{Var} \Theta_{k}<\infty$, where $T_{k}=\tau_{k+1}-\tau_{k}$ is the time needed for the vertical random walk to cross the strip bounded by $z_{k}$ and $z_{k+1}$.

Additionally, $\lim _{n \rightarrow \infty}\left|F_{L, 2 n}\right|=\infty$ a.s., due to the recurrence of the simple symmetric vertical random walk $\left(Y_{k}\right)$. From the weak law of large numbers, it follows that for all $\varepsilon>0$

$$
\lim _{n \rightarrow \infty} \mathbb{P}_{0}\left(\left|\frac{\sum_{k \in F_{L, 2 n}} \Theta_{k}}{\left|F_{L, 2 n}\right|}-\mu\right| \leq \varepsilon\right)=1,
$$

hence for all $\alpha \in] 0,1\left[\right.$ and sufficiently large $n, \mathbb{P}_{0}\left(\left|\frac{\sum_{k \in F_{L, 2 n}} \Theta_{k}}{\left|F_{L, 2 n}\right|}-\mu\right| \leq \varepsilon\right) \geq \alpha$. Since, for $\mathbf{z} \in \operatorname{ConsAdm}(L, 2 n, d)$ we have $\left|F_{L, 2 n}\right| \leq d \sqrt{n}$, we conclude that for all $n$ sufficiently large, $\mathbb{P}_{0}\left(\left|\sum_{k \in F_{L, 2 n}} \theta_{k}\right| \leq d^{\prime} \sqrt{n} \mid \mathscr{G}\right)>\alpha$, with any $d^{\prime}>\mu d$. Finally, for $n$ sufficiently large,

$$
\sum_{\mathbf{z} \in \operatorname{Cons} A d m(L, 2 n, d)} \mathbb{P}_{0}(C[\mathbf{z}] \mid \mathscr{G})=\mathbb{P}_{0}\left(\sum_{z:|z| \leq L+1} \omega(z) \leq d \sqrt{n} \mid Z_{2 n}=0\right) \mathbb{P}_{0}\left(Z_{2 n}=0\right) \geq(1-\delta) \frac{c_{3}}{\sqrt{n}}
$$

from lemma 4.3, where $Z_{k}=Y_{\tau_{k}}$. This concludes the proof of the recurrence.

Proof of the proposition 1.8: Since $\|\boldsymbol{\lambda}\|<\infty$, it follows that there is a positive integer $l$ such that $\lambda_{y}=0$ for all $y$ with $|y|>l$. Choosing then an integer $L>$ $[l / Q]+1$ in the above proof of the recurrence part, we immediately conclude. 


\section{Conclusion, open problems, and further developments}

As was apparent in the course of the proof of recurrence, the condition $\beta>1$ is used only to show that there are almost surely finitely many lines where the periodicity imposed by $f$ is perturbed by a random defect. Therefore this condition can be improved. As a matter of fact, the walk is recurrent provided that there exists an arbitrarily large integer $l$ such that the decay is of the form $c\left(|y| \ln |y| \cdots \ln _{l-1}|y| \ln _{l}^{\beta_{l}}|y|\right)^{-1}$ for some $\beta_{l}>1$ (arbitrarily close to 1), where $\ln _{l}$ is the $l$-times iterated logarithm. Nevertheless, our methods do not allow the treatment of the really critical case $\beta_{0}=1$.

It is easy to build up examples in which the random walk is recurrent although there are infinitely many defects, provided they are sparse. The following deterministic construction illustrates this fact. Let $\left(a_{n}\right)_{n \in \mathbb{N}}$ be an increasing sequence of positive numbers such that $a_{n} \rightarrow \infty$. For an arbitrary $\{0,1\}$-valued sequence $\boldsymbol{\lambda}=\left(\lambda_{y}\right)_{y \in \mathbb{Z}}$ we denote by $\boldsymbol{\lambda} \uparrow_{k}$ its restriction to $\{-k, \ldots, k\}$ (meaning that $\left(\lambda\left\lceil_{k}\right)_{y}=\lambda_{y}\right.$ if $|y| \leq k$ and vanishes otherwise) for $k \in \mathbb{N}$; define also $\lambda \uparrow_{\infty} \equiv \boldsymbol{\lambda}$. Obviously $\left\|\boldsymbol{\lambda} \uparrow_{k}\right\|=\operatorname{card}\left\{y \in \mathbb{Z}:|y| \leq k, \lambda_{y}=1\right\}$. For a given sequence $\boldsymbol{\lambda}$, we write $\mathbb{P}[\boldsymbol{\lambda}]_{0}(\cdot)$ for the probability measure corresponding to the environment $\boldsymbol{\lambda}$. We shall construct iteratively an infinite deterministic sequence $\boldsymbol{\lambda}=\left(\lambda_{y}\right)_{y \in \mathbb{Z}}$ of defects perturbing the periodic sequence determined by $f$ so that the corresponding random walk is recurrent. The first defect is inserted at level 0 , i.e. we initialise the sequence to $\lambda_{y}^{(1)}=\delta_{y, 0}$ so that $\left\|\boldsymbol{\lambda}^{(1)}\right\|=1$. Now the random walk with only one defect in the whole vertical axis is recurrent, meaning that

$$
\sum_{n \in \mathbb{N}} \mathbb{P}\left[\boldsymbol{\lambda}^{(1)}\right]_{0}\left(\mathbf{M}_{n}=(0,0)\right)=\infty .
$$

Therefore there exists a positive integer $L_{1}>0$ such that

$$
\sum_{n=1}^{L_{1}} \mathbb{P}\left[\boldsymbol{\lambda}^{(1)}\right]_{0}\left(\mathbf{M}_{n}=(0,0)\right) \geq a_{1} .
$$

But since in time $L_{1}$, the vertical random walk cannot be further than $L_{1}$ from 0 , nothing changes if instead of choosing the sequence $\boldsymbol{\lambda}^{(1)}$ as above with $\left\|\boldsymbol{\lambda}^{(1)}\right\|=$ 1 , we chose any other sequence $\boldsymbol{\lambda}^{\prime}$ with $\lambda_{0}^{\prime}=\lambda_{0}^{(1)}=1$ and $\left.\left\|\boldsymbol{\lambda}^{\prime} \uparrow_{L_{1}}\right\|\right)=1$ in the above formula. The second defect is inserted at level $L_{1}+1$, i.e. we modify the sequence into a the sequence $\boldsymbol{\lambda}^{(2)}$ verifying $\lambda_{y}^{(2)}=\delta_{y, 0}+\delta_{y, L_{1}+1},\left.\boldsymbol{\lambda}^{(2)}\right|_{L_{1}}=\boldsymbol{\lambda}^{(1)}$, and $\left\|\boldsymbol{\lambda}^{(2)}\right\|=2$. Again, we can choose a positive integer $L_{2}>L_{1}$ such that $\sum_{n=1}^{L_{2}} \mathbb{P}\left[\boldsymbol{\lambda}^{(2)}\right]_{0}\left(\mathbf{M}_{n}=\right.$ $(0,0)) \geq a_{2}$, and so on. We construct in that way a deterministic sequence

$$
\boldsymbol{\lambda}=\lim _{k \rightarrow \infty} \boldsymbol{\lambda}^{(k)} \text { such that } \lambda_{y}=\delta_{y, 0}+\sum_{k=1}^{\infty} \delta_{y, L_{k}+1} \text { for } y \in \mathbb{Z},
$$

verifying (by construction) $\|\boldsymbol{\lambda}\|=\infty$ and $\left\|\boldsymbol{\lambda} \uparrow_{L_{k}}\right\|=k$ for all $k$. For every $k \in \mathbb{N}$, during its first $L_{k}$ steps, the random walk can reach no more than the $k$ first 
defects and for $n \leq L_{k}$, we have therefore $\mathbb{P}[\boldsymbol{\lambda}]_{0}\left(\mathbf{M}_{n}=(0,0)\right)=\mathbb{P}\left[\boldsymbol{\lambda}\left\lceil_{L_{k}}\right]_{0}\left(\mathbf{M}_{n}=\right.\right.$ $(0,0))$. Hence

$$
\begin{aligned}
\sum_{n=0}^{\infty} \mathbb{P}[\boldsymbol{\lambda}]_{0}\left(\mathbf{M}_{n}=(0,0)\right) & \geq \sum_{n=0}^{L_{k}} \mathbb{P}[\boldsymbol{\lambda}]_{0}\left(\mathbf{M}_{n}=(0,0)\right) \\
& =\sum_{n=0}^{L_{k}} \mathbb{P}\left[\boldsymbol{\lambda} \uparrow_{L_{k}}\right]_{0}\left(\mathbf{M}_{n}=(0,0)\right) \\
& \geq a_{k} .
\end{aligned}
$$

Since $k$ is arbitrary, this implies recurrence.

Acknowledgements: The authors should like to thank the referee for the very careful reading and the numerous remarks that helped them improving the presentation of the paper and correcting an erroneous statement in the proof of recurrence.

\section{References}

[1] Rabi Bhattacharya and Edward C. Waymire. A basic course in probability theory. Universitext. Springer, New York, 2007. 10, 12

[2] Norman Biggs. Algebraic graph theory. Cambridge University Press, London, 1974. Cambridge Tracts in Mathematics, No. 67. 3

[3] Massimo Campanino and Dimitri Petritis. Random walks on randomly oriented lattices. Markov Process. Related Fields, 9(3):391-412, 2003. 2, 3, 5, 6, 7

[4] Massimo Campanino and Dimitri Petritis. On the physical relevance of random walks: an example of random walks on a randomly oriented lattice. In Random walks and geometry, pages 393-411. Walter de Gruyter GmbH \& Co. KG, Berlin, 2004. 2

[5] Fan R. K. Chung. Spectral graph theory, volume 92 of CBMS Regional Conference Series in Mathematics. Published for the Conference Board of the Mathematical Sciences, Washington, DC, 1997. 3

[6] Dragoš M. Cvetković, Michael Doob, and Horst Sachs. Spectra of graphs. Johann Ambrosius Barth, Heidelberg, third edition, 1995. Theory and applications. 3

[7] Basile de Loynes. Marche aléatoire sur un di-graphe et frontière de Martin. C. R. Math. Acad. Sci. Paris, 350(1-2):87-90, 2012a. 2

[8] Alexis Devulder and Françoise Pène. Random walk in random environment in a two-dimensional stratified medium with orientations. Electron. J. Probab., 18:no. 18, 23, 2013. 2

[9] Peter G. Doyle and J. Laurie Snell. Random walks and electric networks, volume 22 of Carus Mathematical Monographs. Mathematical Association of America, Washington, DC, 1984. 3

[10] Harley Flanders. Infinite networks. I: Resistive networks. IEEE Trans. Circuit Theory, CT-18:326-331, 1971b. 3

[11] Harley Flanders. Infinite networks. II. Resistance in an infinite grid. J. Math. Anal. Appl., 40:30-35, 1972. 3

[12] N. Guillotin-Plantard and A. Le Ny. Transient random walks on 2D-oriented lattices. Teor. Veroyatn. Primen., 52(4):815-826, 2007. 2 
[13] Nadine Guillotin-Plantard and Arnaud Le Ny. A functional limit theorem for a 2D-random walk with dependent marginals. Electron. Commun. Probab., 13:337-351, 2008. 2

[14] Palle E. T. Jorgensen and Erin P. J Pearse. Operator theory and analysis of infinite networks, 2008. arXiv:0806.3881. 3

[15] G. Matheron and G. de Marsily. Is transport in porous media always diffusive? a counter-example. Water Resour. Res., 16:901-917, 1980. 2

[16] Françoise Pène. Transient random walk in $\mathbb{Z}^{2}$ with stationary orientations. ESAIM Probab. Stat., 13:417436, 2009. 2

[17] Gábor Pete. Corner percolation on $\mathbb{Z}^{2}$ and the square root of 17. Ann. Probab., 36(5):1711-1747, 2008. 2

[18] Sidney C. Port. Theoretical probability for applications. Wiley Series in Probability and Mathematical Statistics: Probability and Mathematical Statistics. John Wiley \& Sons Inc., New York, 1994. A WileyInterscience Publication. 16

[19] Sidney Redner. Survival probability in a random velocity field. Phys. Rev. E, 56:4967-4972, 1997. 2

[20] Paolo M. Soardi. Potential theory on infinite networks, volume 1590 of Lecture Notes in Mathematics. Springer-Verlag, Berlin, 1994. 3

[21] Hermann Weyl. Repartición de corriente en una red conductora. Revista Matemática HispanoAmericana, 5:153-164, 1923. 3

[22] Wolfgang Woess. Random walks on infinite graphs and groups, volume 138 of Cambridge Tracts in Mathematics. Cambridge University Press, Cambridge, 2000. 3 